\title{
Zur Kenntnis der Korksubstanz
}

\author{
(III. Mitteilung) \\ von \\ M. v. Schmidt.
}

Aus dem Laboratorium für analytische Chemie der $k$. $k$. Hochschule für Bodenkultur.

(Vorgelegt in der Sitzung am 3. März 1910.)

\section{Wesen und Synthese der Korksubstanz.}

In einer früheren Mitteilung ${ }^{1}$ habe ich auf die bemerkenswerte Tatsache hingewiesen, daß Korkmehl an Chloroform, Benzol und ähnliche indifferente Lösungsmittel, neben geringen Mengen eines $\gg$ Cerin $\ll$ genannten aromatischen Alkohols, zirka $10 \%$ Glyceride abgibt, während alkoholisches Kali hierauf noch weitere $30 \%$ Fettsäuren, aber kein Glycerin zu extrahieren vermag.

Daraus muß gefolgert werden, daß die Hauptmenge der Korkfettsäuren in den Suberinlamellen der Zellwand nicht als Glycerinester, sondern in irgendeiner anderen, und zwar unlöslichen Form auftritt.

Der Gedanke liegt nahe, sie an Cerin gebunden zu denken. Aber abgesehen davon, daß die Cerinkrystalle stets isoliert im Zellinnern angetroffen werden, müßten derartige wachsähnliche Verbindungen doch irgendwie zu lösen sein. Das Cerin selbst und ebenso die hier vorhandenen Fettsäuren lösen sich ziemlich leicht; unlösliche Wachsarten sind bis jetzt überhaupt nicht aufgefunden worden. Wären aber solche dennoch vorhanden, so müßte das bei der Verseifung entstehende Cerin in die

1 Sitzungsber. der Wiener Akademie, Bd. CXII, Abt. II b, Dezember 1903. 
alkoholische Lösung übergehen. Dies ist aber erfahrungsgemäß nicht der Fall. Unter der Voraussetzung, daß der Kork vor der Behandlung mit alkoholischem Kali durch Chloroform oder Benzol vollständig erschöpft wird, geben die erhaltenen Seifen an Äthter und ähnliche Solventien weder Cerin, noch irgendeinen anderen alkoholähnlichen Stoff $a b$.

Es bleibt sonach nur die Annahme übrig, daß die Fettsäuren des Korkes in Gestalt von Anhydriden oder Polymerisationsprodulten vorhanden sind, die ganz wohl unlöslich, aber verseifbar sein können.

Um die Möglichkeit einer derartigen Anhydridbildung experimentell nachzuweisen, versuchte ich das Verhalten der einzigen bis jetzt genauer studierten Fettsäure des Korkes, der Phellonsäure, gegen höhere Temperaturen festzustellen. Das Erhitzen wurde im Kohlensäurestrome vorgenommen und der Versuch derart angeordnet, daß sowohl das abgespaltene Wasser, als auch das Reaktionsprodukt, gewogen werden konnte.

Zur Aufnahme der in ein Schiffchen eingewogenen Säure diente ein beiderseits offenes Rohr aus Kaliglas, welches in ein doppelwandiges Kupferrohr eingesetzt wurde, in dessen Mantelraum Xyloldampf zirkulierte. Ein seitliches Ansatzrohr dieses letzteren war zur Aufnahme des Thermometers bestimmt. Die vom Beginn des Versuches an durch den Apparat geleitete Kohlensäure war über Chlorcalcium getrocknet und führte die Wasserdämpfe in ein gewogenes Chlorcalciumrohr, an welches vorsichtshalber ein zweites angeschlossen wurde. Die Temperatur des Xyloldampfes schwankte zwischen 139 und $142^{\circ}$. $\mathrm{Da}$ ich über die Zeitdauer der möglichen Anhydridbildung völlig im unklaren war, wurde die Operation von $6 \mathrm{zu} 6$ Stunden unterbrochen, um den Gewichtsverlust der Probe zu ermitteln.

Abgewogen wurden $0.3338 \mathrm{~g}$ Phellonsäure, welche samt dem Schiffchen $4 \cdot 3617 g$ repräsentierten. Dieses Gewicht sank nach 6 Stunden auf $4 \cdot 3576 \mathrm{~g}$

\begin{tabular}{|c|c|c|}
\hline & 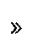 & \\
\hline & 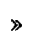 & \\
\hline & » & \\
\hline & " & \\
\hline
\end{tabular}




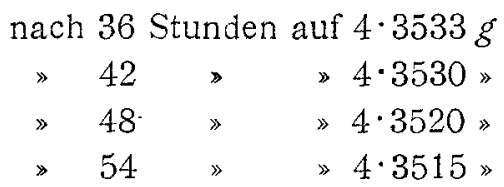

und blieb dann konstant.

Der Gesamtgewichtsverlust betrug sonach $0.0102 \mathrm{~g}=$ $3.05 \%$. Daß er auf Wasserabspaltung zurückzuführen war, ergab sich aus der Gewichtszunahme des Chlorcalciumrohres, die damit fast vollkommen übereinstimmte. Für den Austritt von einem Molekül Wasser berechnet sich ein Gewichtsverlust von $2 \cdot 54 \%$. Die Differenz zwischen dieser Zahl und der gefundenen erscheint auf den ersten Blick bedeutend. Da sie aber, absolut genommen, nur $2 \mathrm{mg}$ ausmacht, so wird sie durch Versuchsfehler leicht erklärt. Zum Überflusse wurde das Anhydrid der Elementaranalyse unterworfen, welche folgende Werte ergab.

$0.3018 g$ Substanz gaben $0.8450 \mathrm{~g}$ Kohlensäure und $0.2867 \mathrm{~g}$ Wasser.

In 100 Teilen:

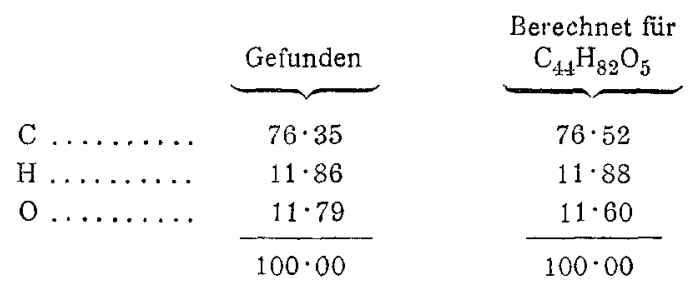

Das so erzeugte Anhydrid war im Aussehen nicht merklich verschieden von der Säure. Es bildete eine harte, spröde, deutlich krystallinische Masse, die bei $102^{\circ}$ schmolz. In Alkohol und Petroleumäther ist es so gut wie unlöslich, wird aber von kochendem Chloroform, Benzol, Eisessig und Pyridin ziemlich leicht gelöst, so daß es ohne Schwierigkeit daraus umkrystallisiert werden kann. Durch wässerige Lauge ist es nur schwierig, durch alkoholische in der Siedehitze leicht verseifbar.

Die Leichtigkeit, mit welcher Phellonsäure Wasser abzuspalten vermag, veranlaßte mich zu versuchen, ob dies unter günstigeren Bedingungen nicht auch bei niedrigerer Temperatur 
$z u$ erzielen wäre. $1 \mathrm{~g}$ derselben wurde mit $10 \mathrm{~cm}^{3}$ rauchender Salzsäure in ein Glasrohr eingeschlossen und dieses 6 Stunden lang im Wasserbade erhitzt. Hierauf wurde der von der Säure getrennte Krystallkuchen mit Wasser mehrmals umgeschmolzen und aus Benzol umkrystallisiert. Es gelang leicht, die so erhaltenen Krystalle durch ihren Schmelzpunkt $\left(102^{\circ}\right)$ und ihre Löslichkeitsverhältnisse mit dem auf trockenem Wege erzeugten Anhydrid zu identifizieren.

$\mathrm{Da}$ die Möglichkeit nicht ausgeschlossen war, durch Einwirkung höherer Temperaturen Anhydride von anderer Zusammensetzung zu erhalten, so wurde bei einem dritten Versuche Phellonsäure auf $180^{\circ}$ erhitzt. Es zeigte sich aber, daß unter diesen Umständen auch bei Abschluß von Luft bereits eine tiefer greifende Veränderung eintritt. Die Masse färbt sich braun und hat ihre krystallinische Beschaffenheit, ihre Schmelzbarkeit und ihre Löslichkeit in allen indifferenten Solventien eingebüßt. Das Wesen dieser Veränderung zu ergründen, reichte mein bescheidener Vorrat an Substanz nicht hin. Sie ist, als unter abnormen Bedingungen entstanden, auch irrelevant für die Konklusionen, die sich an die bei niedrigerer Temperatur erfolgende glatte Wasserabspaltung knüpfen lassen.

Die große Neigung der Phellonsäure, Anhydride zu bilden, spricht sehr zugunsten der Vermutung, daß sie im Kork hauptsächlich in dieser Form enthalten ist. Denn ein Prozeß, der bei $140^{\circ}$ in 54 Stunden quantitativ verläuft, kann bei gewöhnlicher Temperatur im Verlaufe von Jahren mindestens partiell sich abspielen. In Widerspruch damit steht allerdings die Tatsache, daß das an und für sich in Chloroform und Benzol leicht lösliche Anhydrid als solches überhaupt nicht, sondern erst nach erfolgter Verseifung in Gestalt des Kalisalzes der Säure aus dem Kork extrahiert werden kann. Dieser Widerspruch ließe sich durch die Annahme erklären, daß die neben Phellonsäure vorhandenen Korkfettsäuren eine ähnliche Umwandlung erlitten haben und dabei in Formen übergingen, die zwar verseifbar sind, aber allen indifferenten Lösungsmitteln widerstehen. So wäre es verständlich, daß das darin eingebettete Anhydrid der Phellonsäure der Einwirkung dieser Agenzien entzogen wird, wie denn z. B. auch eingetrocknetes 
Leinöl an Benzol nichts abgibt, trotzdem Stearinsäure, Palmitinsäure und andere lösliche Fettsäuren darin enthalten sind.

Ist diese Erklärung zutreffend, so muß das Gemisch der rohen Fettsäuren des Korkes beim Erhitzen gleichfalls ein unlösliches Produkt liefern.

Um dies festzustellen, wurden einige Gramm desselben, wie oben beschrieben, irn Kohlensäurestrome 6 Stunden lang auf $140^{\circ}$ erhitzt. Auch hierbei wurde ersichtlich Wasser abgespalten, auf dessen Wägung aus naheliegenden Gründen verzichtet werden mußte. Das so entstandene Produkt erwies sich als eine braun gefärbte, elastische, durchsichtige Masse ohne Spur von krystallinischem Gefüge, aus welcher tatsächlich durch kein wie immer geartetes indifferentes Lösungmittel irgend etwas ausgezogen werden konnte. Diese veränderten Fettsäuren erweichen in der Hitze nicht mehr, sind impermeabel für Gase und repräsentieren die eigentliche Korksubstanz. Ein mit dem rohen Fettsäuregemisch imprägniertes Filtrierpapier verhält sich nach kurzem Erhitzen auf $140^{\circ}$ wie eine dünne Schnitte Kork. Verschließt man damit das eine Ende eines U-Rohres und füllt Quecksilber ein, so daß in dem offenen Schenkel ein Überdruck von einigen Zentimetern herrscht, so wird auch nach Jahr und Tag die Niveaudifferenz nicht ausgeglichen. Noch mehr springt die Identität dieser Masse mit Kork in die Augen, wenn man in die geschmolzenen Fettsäuren ein gleiches Gewicht Holzmehl einrührt, aus dem Brei einen Stöpsel formt und diesen im Luftbade auf 140 bis $150^{\circ}$ längere Zeit erhitzt. Es resultiert ein Gebilde, das in bezug auf Farbe, Elastizität und Verarbeitungsmöglichkeit täuschend an Kork erinnert und nur durch den Mangel der charakteristischen Struktur von diesem verschieden ist. Man kann es schneiden, feilen und bohren, es erweist sich als undurchdringlich für Wasser und Gase, hat fast dasselbe spezifische Gewicht wie Kork und wird von keinem Lösungsmittel, außer alkoholischem Kali, angegriffen. Bei der Oxydation mit Salpetersäure liefert es Korksäure und Oxalsäure, an der Luft entzündet, brennt es mit stark rußender Flamme unter Verbreitung des charakteristischen Geruches von echtem Kork. Man hat es hier tatsächlich mit einer Art Synthese, mit einer Regeneration der Korksubstanz 
aus ihren Verseifungsprodukten $\mathrm{zu}$ tun. Einen stringenten Beweis dafür, daß diese Umwandlung der Fettsäuren auch bei gewöhnlicher Temperatur vor sich gehen kann, vermochte ich allerdings nicht zu erbringen. Es gelang mir aber, sie bei $120^{\circ}$ im Verlaufe von 24 Stunden vollständig, bei $100^{\circ}$ in 48 Stunden zum großen Teile, bei $50^{\circ}$ in 72 Stunden in nachweisbarer Menge herbeizuführen, so daß ihr Vollzug bei gewöhnlicher Temperatur im Verlaufe von Jahren mehr als wahrscheinlich erscheint. Erwägt man, daß die freien Fettsäuren trocknender Öle um vieles langsamer in feste Verbindungen übergehen als ihre Glyceride, so darf es nicht wundern, wenn meine gleichfalls mit Fettsäuren angestellten Versuche der Intervention von Wärme bedurften, um in absehbarer Zeit ein greifbares Ergebnis zu liefern.

Den Hauptanteil an der Umwandlung der Korkfettsäuren in die unlösliche Form nimmt die in größter Menge vorhandene, şchon von Gils on erwähnte flüssige Suberinsäure, die ich zwar noch nicht analysenrein, aber dank ihrer Unlöslichkeit in Petroleumäther frei von festen Fettsäuren isolieren konnte. Sie scheint ungesättigt $z u$ sein und verwandelt sich beim Erhitzen in eine vollkommen unlösliche, elastische, eingetrocknetem Leinöl nicht unähnliche Masse. $\mathrm{Da}$ die Umwandlung dieser Säure ohne Wasserabspaltung erfolgt und - als bei Luftabschluß eingetreten - auch nicht auf Oxydation zurückgefühtt werden kann, so liegt hier ohne $Z_{w}$ eifel Polymerisation vor. Tatsächlich ergab die Elementaranalyse der in den festen Zustand übergeführten Säure dieselben Zahlen wie die unmittelbar aus Kork gewonnene flüssige. Ich unterlasse es vorläufig, die erhaltenen Werte anzuführen, weil sie im Hinblick auf die noch nicht völlig sichergestellte Reinheit der Substanzen nur relative Geltung haben. Gewiß ist nur, daß diese Säure, ähnlich der Eläomargarinsäure des Holzöles bei dem Übergang in die feste Modifikation ihre prozentische Zusammensetzung nicht ändert.

Nach dem Gesagten ist das Vorhandensein von Fettsäuren im Korke, die durch keinerlei Lösungsmittel daraus extrahiert werden können, nicht mehr rätselhaft. Da neben diesen auch leicht ausziehbare Glyceride in geringer Menge zugegen sind, so drängt sich die Vermutung auf, daß ursprünglich die Gesamt- 
menge der Fettsäuren an Glycerin gebunden war. Unter dem Einflusse von Luft, Licht und vielleicht auch durch Enzymwirkung, mußte dann eine partielle Spaltung der Glycerinester eingetreten sein, ähnlich jener, wie wir sie bei trocknenden Ölen beobachten. Das Glycerin wurde zu Kohlensäure und Wasser oxydiert, die Fettsäuren teils polymerisiert, teils in Anhydride verwandelt und dadurch in den unlöslichen Zustand übergeführt. Was also durch Lösungsmittel dem Kork entzogen werden kann, ist neben Cerin jener Rest von ungespaltenen Glyceriden; was durch alkoholisches Kali verseift wird, ein Gemisch von Säureanhydriden und Polymerisationsprodukten, welche Mutmaßung mit der von mir konstatierten Abwesenheit des Glycerins in dieser Lösung im Einklang steht.

Die hier erläuterte Bildung der Korksubstanz hat mit Rücksicht auf die mitgeteilten Tatsachen große Wahrscheinlichkeit für sich. Als einwandfrei bewiesen kann sie aber erst dann betrachtet werden, wenn es gelingt, den Nachweis dafür zu erbringen,

1. daß die durch Lösungsmittel extrahierbaren Glycerinester des Korkes dieselben Fettsäuren enthalten wie die durch alkoholisches Kali daraus gewonnenen Seifen;

2. daß auch diese Glyceride unter geeigneten Umständen in unlösliche Modifikationen übergeführt werden können;

3. daß durch Verseifung der künstlich erzeugten unlöslichen Produkte sich gleichfalls lösliche Fettsäuren regenerieren lassen.

Die erste dieser Fragen ist bereits von Kügler und übereinstimmend mit ihm von mir, in bejahendem Sinne beantwortet worden. Ich fand Phellonsäure neben anderen, noch nicht näher untersuchten, sowohl in dieser als in jener Fraktion; nur Suberinsäure war im Chloroformextrakt des Korkes nicht nachzuweisen.

Die Aufklärung des zweiten strittigen Punktes begegnet wegen der Unmöglichkeit, die im Kork präformierten Glycerinester von dem gleichzeitig extrahierten Cerin zu trennen, großen Schwierigkeiten. Wird der eingedunstete Chloroformextrakt des Korkes längere Zeit auf $180^{\circ}$ erhitzt, so verliert er seine Löslichkeit nur partiell. Cerin läßt sich mit Leichtigkeit daraus ausziehen und was zurückbleibt, erscheint nicht als zusammen- 
hängende elastische Masse, sondern tritt in bräunlichen Flocken auf, die auch nicht zusammengeschmolzen werden können. Der Versuch erschien also, in dieser Form ausgeführt, nicht konkludent; wohl aber gelang er mit den künstlich hergestellten Glyceriden der Korkfettsäuren.

Das Gemisch roher Fettsäuren, welches durch Verseifung des mit Chloroform erschöpften Korkes erhalten war, wurde mit überschüssigem Glycerin unter Durchleiten von Kohlensäure auf $200^{\circ}$ erhitzt. Unter diesen Umständen tritt leicht Veresterung ein. Schon nach Verlauf einer Viertelstunde konnte eine herausgezogene Probe durch Natriumcarbonat nicht mehr verseift werden, ein Beweis, daß die Umwandlung in Glyceride vollendet war. Sonach wurde das Reaktionsprodukt vom Glycerin getrennt, mit Wasser wiederholt umgeschmolzen und getrocknet. Es verhielt sich beim Erhitzen auf $180^{\circ}$ genau wie die Säuren selbst: die Masse erweichte in der Wärme nicht mehr, hatte die krystallinische Beschaffenheit eingebüßt und erwies sich als vollkommen unlöslich in Chloroform, Benzol, Eisessig u. dgl.

Es blieb sonach nur noch die Frage zu erledigen, ob durch Behandlung dieser künstlich dargestellten Korksubstanz mit alkoholischem Kali die ursprünglichen Fettsäuren zurückgebildet werden können. Daß hierbei leicht Verseifung eintrat und auf Zusatz von Salzsäure neuerdings Fettsäuren ausgeschieden wurden, war leicht $z u$ beobachten. Ob diese aber identisch mit der Muttersubstanz waren, konnte in kurzem Wege nicht anders als durch das Ansehen und den beiläufigen Schmelzpunkt beurteilt und bejaht werden. Eine Trennung der zurïckgebildeten Fettsäuren war nur mit enormem Aufwand an Zeit und Versuchsmaterial zu erreichen; ich hielt es daher für einfacher, den Versuch, von reinen Substanzen ausgehend, $z \mathrm{u}$ wiederholen. Sonach verseifte ich Phellonsäureanhydrid, zersetzte die alkoholische Seifenlösung mit Salzsäure und verdünnte stark mit Wasser. Die ausfallenden Flocken wurden mit Wasser gewaschen, getrocknet und aus Benzol umkrystallisiert. Die so gereinigte Säure zeigte den Schmelzpunkt $96^{\circ}$, verhielt sich Lösungsmitteln gegenüber wie Phellonsäure und bildete, wie diese, die charakteristischen wasserunlöslichen Alkalisalze. 
In ähnlicher Weise konnte auch aus der durch Erhitzen polymerisierten Suberinsäure die Muttersubstanz regeneriert werden, womit die Beweiskette geschlossen war.

Die Möglichkeit, daß die von mir künstlich dargestellte Korksubstanz nicht absolut identisch mit der natürlich vorkommenden ist, muß zugestanden werden, da jene unter dem Einfluß von Wärme in kurzer Zeit, diese aber bei gewöhnlicher Temperatur allmählich entstanden war.

Wie aber bei gewöhnlicher Temperatur eingetrocknetes Leinöl, trotz seines anderen Aussehens und seiner etwas abweichenden Zusammensetzung, im großen und ganzen doch denselben Stoff darstellt, wie das durch Erhitzen im Luftstrom erzeugte Produkt, so stimmt auch die regenerierte Korksubstanz rücksichtlich ihrer typischen Bestandteile und aller wesentlichen Eigenschaften mit der in der Natur vorkommenden vollkommen überein.

Damit ist ist die Frage nach der Form, in der die Fettsäuren im Kork auftreten, endgültig gelöst und der Hauptzweck meiner dornenvollen Arbeit erreicht. Die nähere Untersuchung der neben Phellonsäure vorhandenen Säuren - es sind ihrer, wie ich heute schon behaupten kann, mindestens noch drei wird voraussichtlich noch längere Zeit in Anspruch nehmen. Doch tritt dieser Teil der Untersuchung voliständig in den Hintergrund gegenüber dem bereits Erreichten: das fast unlösbar scheinende Problem vom Wesen der Korksubstanz, trotz der Unmöglichkeit, sie in nativem Zustande zu isolieren, auf Umwegen ergrünḍet und diese selbst aus ihren Bruchstücken aufgebaut zu haben.

\section{Zusammenfassung.}

Vom Standpunkte des Chemikers betrachtet, ist Korksubstanz ein unlösliches Gemenge von Anhydriden und Polymerisationsprodukten fester und flüssiger Fettsäuren mit Resten von Glycerinestern derselben Säuren. Der junge Kork enthält höchstwahrscheinlich nur Glyceride, die gleich den trocknenden Ölen unter Spaltung und Glycerinverlust allmählich in die erstgenannten Verbindungen übergehen. 
Ich kann meine Arbeit nicht beschließen, ohne eines Forschers zu gedenken, dessen grundlegende Versuche sie angeregt und ermöglicht haben. Es war - seltsam genug ein Botaniker, F. v. Höhnel, der dem Chemiker den Weg wies, die Natur der Korksubstanz mit den Hilfsmitteln des Laboratoriums zu studieren, die er an der Hand mikrochemischer Reaktionen schon vor 30 Jahren richtig erkannt hatte. 